\title{
IMPLEMENTATION OF NEW TECHNOLOGIES IN TRADITIONAL HANDCRAFTS: EXAMPLES FROM THE HIGHER EDUCATION
}

\author{
Souliotou AZ* \\ International Hellenic University, Greece \\ University of Western Macedonia, Greece
}

\begin{abstract}
The implementation of new technologies in traditional handcrafts is being an increasingly popular interdisciplinary practice in arts, education and other aspects of life including entertainment, interior and public design. The present research explores the possibilities of the implementation of electrical and light technologies in traditional handcrafts with the purpose of providing a rich multisensory experience. The implied methodology involves practice-based research through creation of handcrafts with implementation of new technologies by the author-instructor and students in the Higher Education. Students' handmade creations involve a wide range of media: paintings, sculptures, collage, puppets and other constructions with implemented new technologies including light effects, coding, movement sensors and renewable energy in contexts of art, education, interior and public space. The implementation of new technologies turned out to be a hands-on art-and-technology practice with multiple benefits in education. Students realised that many different aspects of the traditional handcrafts were revealed with the use of new technologies and that their implementation brings traditional handcrafts to (another) life. Furthermore, it became clear that students' practice through the implementation of new technologies in traditional handcrafts constitutes their own preparation for applying these technologies to children's handmade constructions and, thus, it is possible to enhance and enrich children's creative experience. The stimulation of many senses thanks to the implementation of new technologies can also improve visual and multisensory culture, foster creativity and contribute to cultural and technological literacy. The tension between the traditional and the contemporary through the coexistence of different media proved to be a fruitful synergy making the final whole more interesting than the sum of its parts.
\end{abstract}

Keywords: art, technology, handcrafts, education, design

\section{Introduction}

The implementation of new technologies in traditional handcrafts is being an increasingly popular interdisciplinary practice in arts, education and other aspects of life including entertainment, interior and public design. The present research explores the possibilities of the implementation of electrical and light technologies in traditional handcrafts with the purpose of providing a rich multisensory experience which, in turn, will contribute to (inter)cultural and technological literacy.

The examples presented in this paper were created by the author-instructor and students from Early Childhood Education Departments and Departments of Visual and Applied Arts in Hellenic Universities. 
The implied methodology involved practice-based research through creation of handcrafts with implementation of electrical and light technologies, as well as reflection and conversation in theory and practice about the creative process and the aesthetic and learning outcomes. As far as the teaching and learning methodology is concerned, it involved demonstrations and hands-on experiential and experimental learning for both the artistic and the technological part of the practice.

Implementation of electrical and light technologies in traditional handcrafts is inscribed in STEAM (Science, Technology, Engineering, Arts \& Mathematics) approach with a strong presence of the letter A (Stroud \& Baines, 2019: 1). However, the practice presented in this research is not constrained to the STEAM model, but instead it also embraces cultural heritage and ethnography, since it refers to symbols, traditions and techniques which originate from Chinese, Japanese and Greek civilizations with the implying perspective of expanding this kind of practice to more civilizations.

The present paper analyses the implementation of electrical technologies in traditional handcrafts in terms of education, but also in terms of art and technology, in order to provide a spherical view as well as a deeper understanding of the ontology of this practice.

The word 'traditional' is a derivative of tradition and indicates a belief or principle that a certain group of people follow for a long time (Cambridge Dictionary, 2020) and pass it through ages (Merriam-Webster, 2020). The meaning of traditional art is twofold: it refers to cultural skills and knowledge of a certain group of people which is passed through generations; it also refers to art made with physical and handcrafted media in opposition with digital art. (Wiktionary, 2020)

The compound word 'technology' is composed of the two Greek words $\tau \dot{\varepsilon} \chi v \eta+\lambda o$ ' $o \varsigma$ (transliterated as techne + logos) indicating the art, skill, craft or any other means of creation (techne) and the word, expression, reason or thought (logos). (Funk, 2020) Contrary to anyone's expectations in the contemporary condition, where technology is a fast-developing field mainly associated with machines, the origins of technology relate closely with the skill of the hand, which is indicated in the word techne. (Latimer, 2017: 645)

The above definitions and etymologies prove that handcrafts, which are objects made with the hands, relate closely with both traditional and technological practices.

Most of the examples of this paper are assemblages that combine traditional materials with new technologies. Assemblages consist of diverse coexisting parts and produce effects that emerge from the whole rather than from the sum of the forces of every element alone (Bennett, 2014: 84). The implementation of new technologies in traditional handcrafts assembles not only diverse materials, but also different times, space-time relations and contexts. The uniqueness and originality of each handcraft lies upon its tradition, i.e. its history of ritual function in the location of its first use (Benjamin, 1973). The implementation of new technologies in each traditional handcraft connects its tradition with the aesthetics and functions of contemporary technologies.

The use of traditional practices and pre-existing symbols and techniques connects the present research also with the appropriation/adaptation which is very much used in Contemporary Art since 1980 and 
refers to the reuse and slight transformation of objects and images that already exist (TATE, 2020) in a way that brings old objects to a new context (Verwoert, 2007).

\section{Traditional Handcrafts \& New Technologies in Higher Education}

The examples presented below were created by the author and students of Early Childhood Educations Departments and Departments of Visual \& Applied Arts in Greece.

Figure 1 shows a sample of Jiănzhǐ Chinese paper cutting technique with symmetrical geometric and semi-geometric patterns formed in Chinese red (paper part) and cut-out holes. The shapes of the holes were transferred on an illuminated board with the use of a LED pen and stencil technique. Students were very much impressed by the aesthetic result and by the different effects of light and colour generated with new technologies through the creative exploitation of the opacity and transparency of the initial handcraft's positive and negative space.
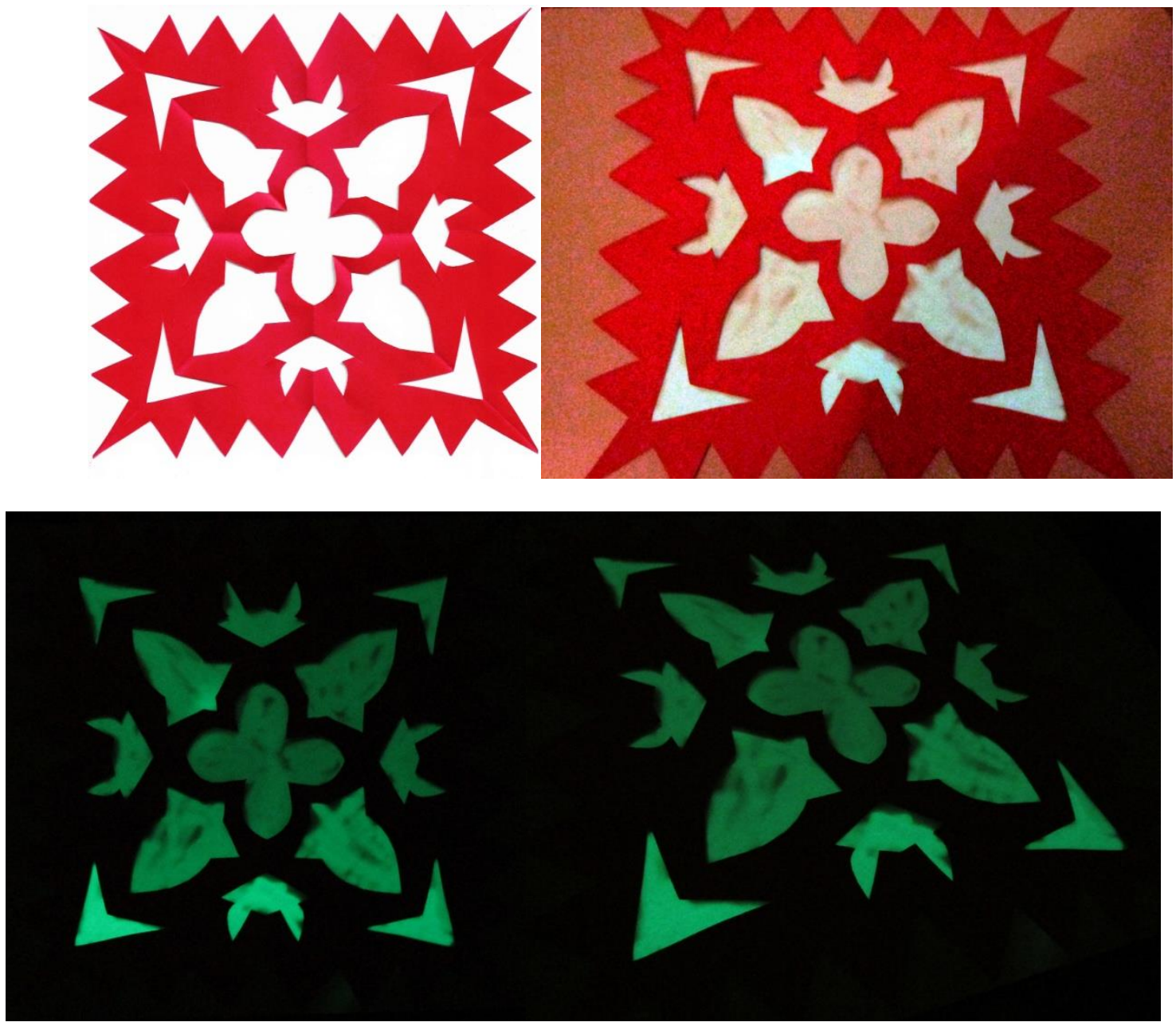

Figure 1 : Sample of Jiănzhǐ Chinese paper cutting technique (up left) and pattern transfer (up right) on an

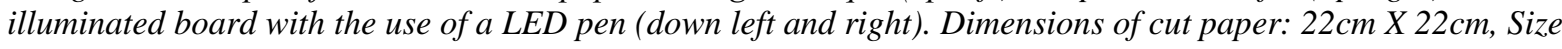
of illuminated board: A3

Another handcraft of Jiănzhǐ Chinese paper cutting technique was wrapped on an RC flying ball (Figure 2). Further than being a mere decorative embellishment of the ball, the cut paper also had the use of making the flying ball more distinguishable in public space during the day especially with the contrast of the Chinese red with the green of the trees and the lawn. At the same time the cut paper did 
not cover the whole ball, but instead it had holes which permit to see and feel the initial texture of the ball.

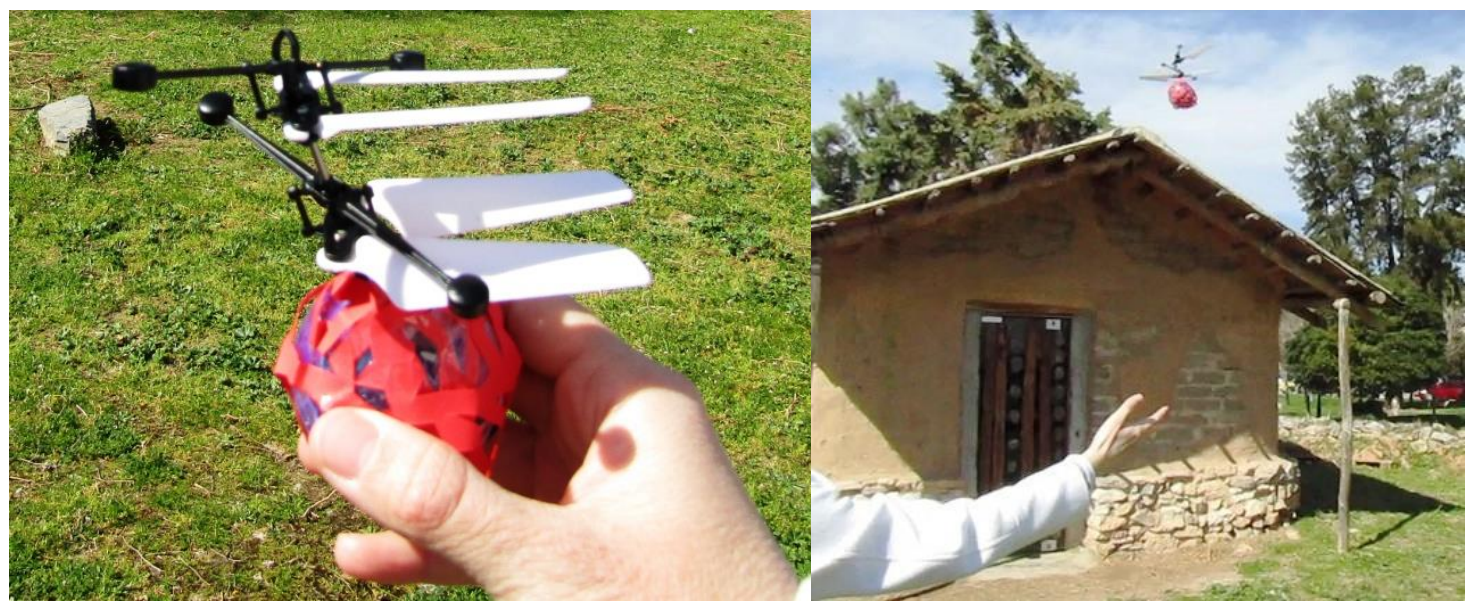

Figure 2: Sample of Jiănzhř Chinese paper cutting technique wrapped on an RC flying ball (left) which flies (right). Dimensions of the RC flying ball: $16 \mathrm{~cm} X 12 \mathrm{~cm}$, Sphere's diameter: $5 \mathrm{~cm}$

Figure 3 presents a table lamp 'dressed' with colourful paper and transformed into calla lily flower with kirigami Japanese technique. It is clear that this activity shows how an everyday object can be creatively transformed with simple materials through kirigami handcrafts. This transformation can have possible applications in interior design, stage design and everyday life.

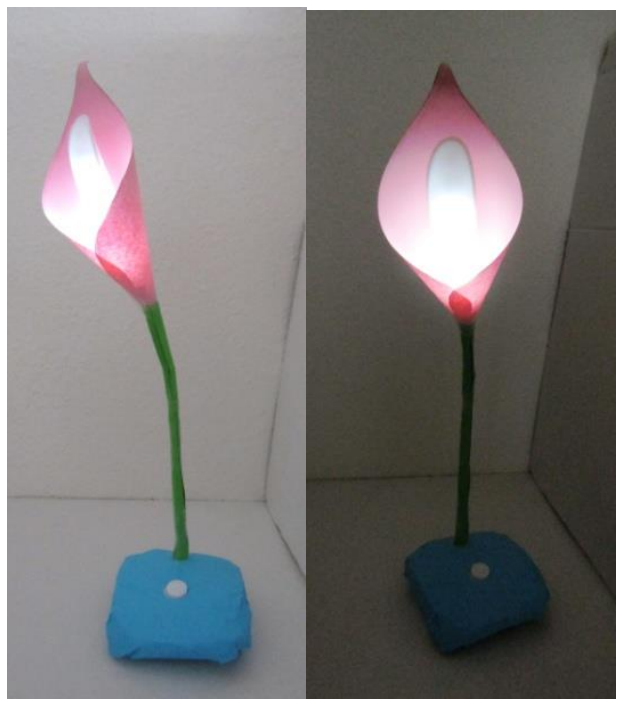

Figure 3: Kirigami colourful paper wrapped on a table lamp, $7 \mathrm{~cm} X 30 \mathrm{~cm} X 7 \mathrm{~cm}$.

Blue Parthenon (Figure 4) is composed of a clay handcraft and a cord of blue neon light which develops freely around the handcraft. This piece refers to the Parthenon temple built on the Acropolis of Ancient Athens. Nowadays Parthenon is a very famous monument and is considered the finest building characterised by harmony and other interesting architectural values which render it a very strong symbol of the Ancient Greek civilization. Despite the handcraft's inaccuracies in Figure 4 and despite its not being an exact copy of Parthenon in a small scale, the reference to the famous ancient Greek temple is clear and inscribes Blue Parthenon in appropriation practices. The combination of a 
handcraft which refers to an ancient monument with neon light creates a new kind of 'ambiance' which dynamically connects the ancient with the modern.

Through art-and-technology practice which combines traditional handcrafts with neon technology adults, students, children and other audiences can come up with interesting aesthetic results, since neon light is a workable and pliable material which creates a wide range of forms (even letters and words) as well as light environments with romantic, evocative or ambient sense.

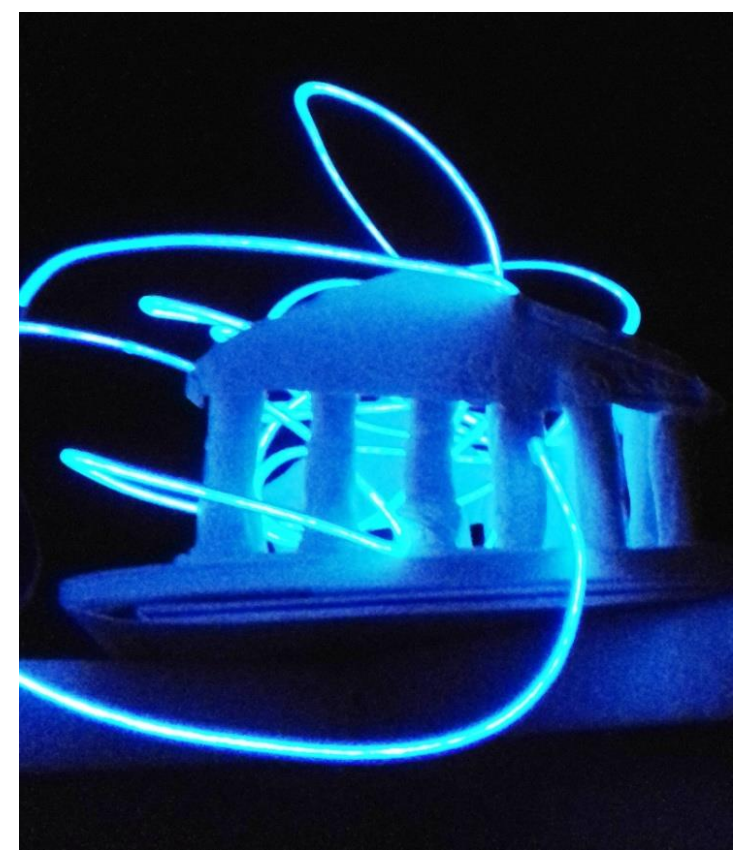

Figure 4: Blue Parthenon, clay handcraft with blue neon light. Dimensions of Parthenon handcraft: $15 \mathrm{~cm} \mathrm{X}$ $7 \mathrm{~cm} \times 5 \mathrm{~cm}$.

Figure 5 shows a hand-painted Styrofoam sculpture with light sensor. The interactivity of the piece enhances the bodily experience of the viewer and simultaneously increases playfulness through the stimulation of visual, spatial, kinaesthetic and even aural perception. The viewer feels more strongly their own presence and this provides an interesting experience both for adults' and children's audiences. 


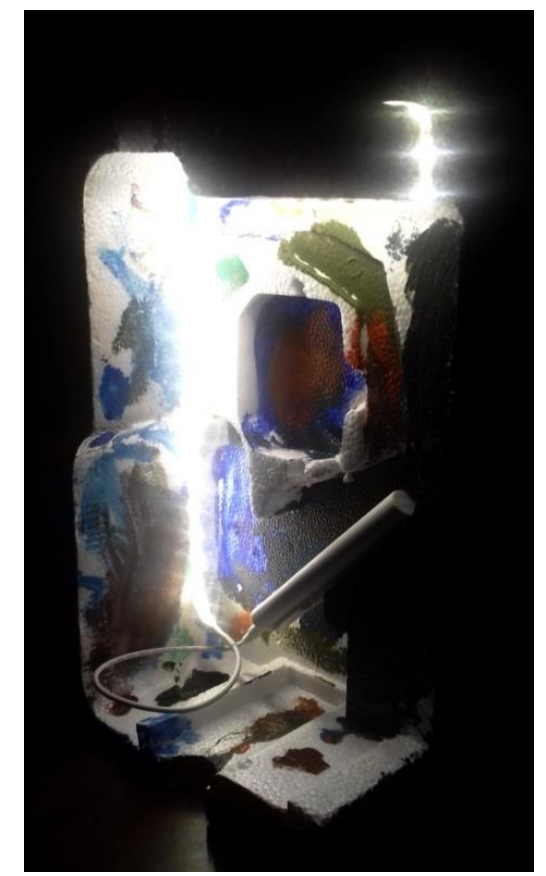

Figure 5: Hand-painted Styrofoam sculpture with light sensor, $22 \mathrm{~cm} \mathrm{X} 36 \mathrm{~cm} \mathrm{X} 12 \mathrm{~cm}$.

Yellow and Non-Yellow Submarine is a single paper construction of an initially yellow submarine which becomes subject to light and colour transformation through the use of a LED tape. The resulting continuous light and colour change creates many different momentary 'ambiances' recalling Guy Debord's vision of the construction of situations (Debord, 1958). This kind of practice can have possible applications in theatre (including small scale puppet theatre and theatre of objects) and stage design as well as in contexts of interior design and children's, adults' and life-long education.
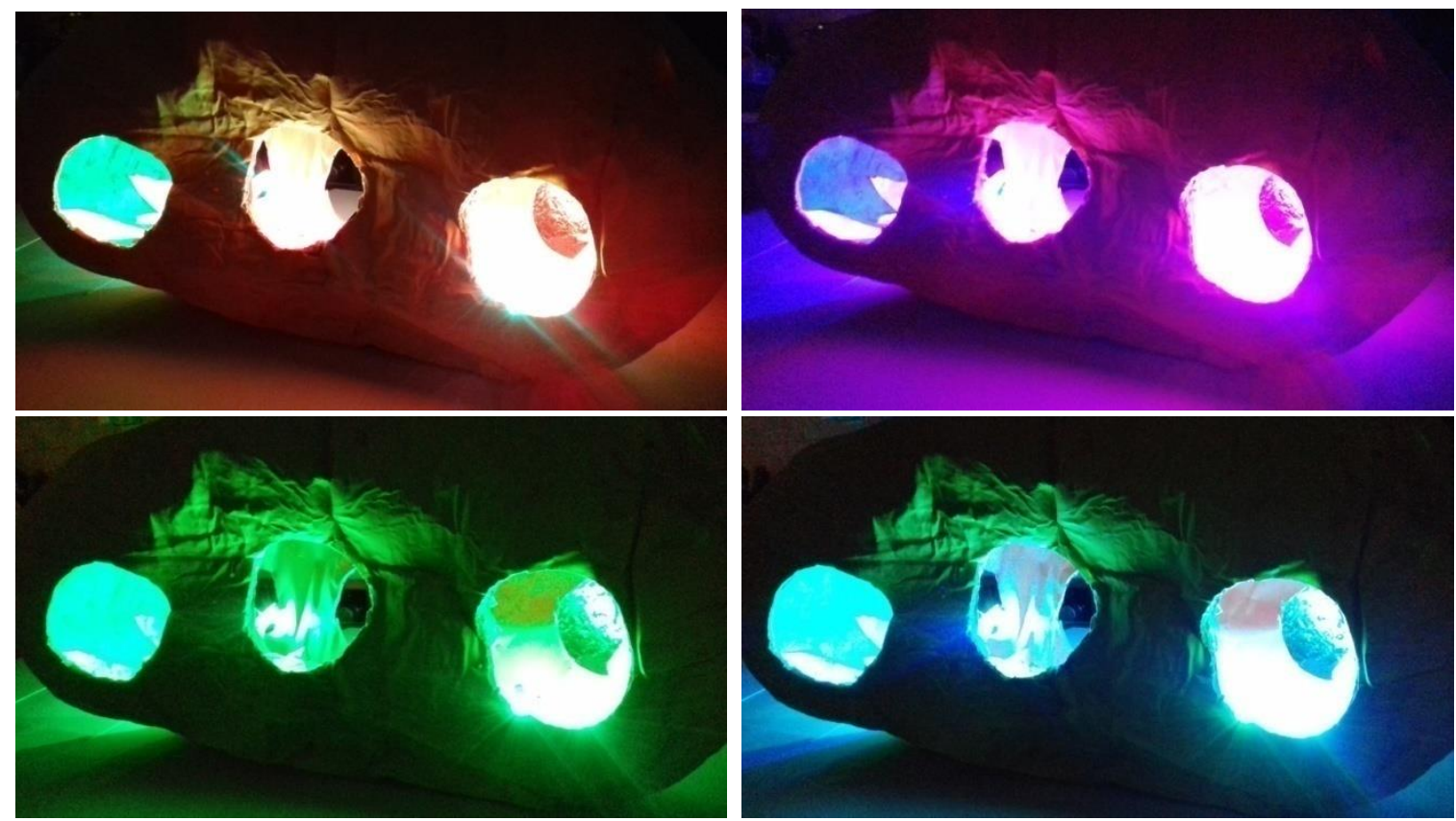

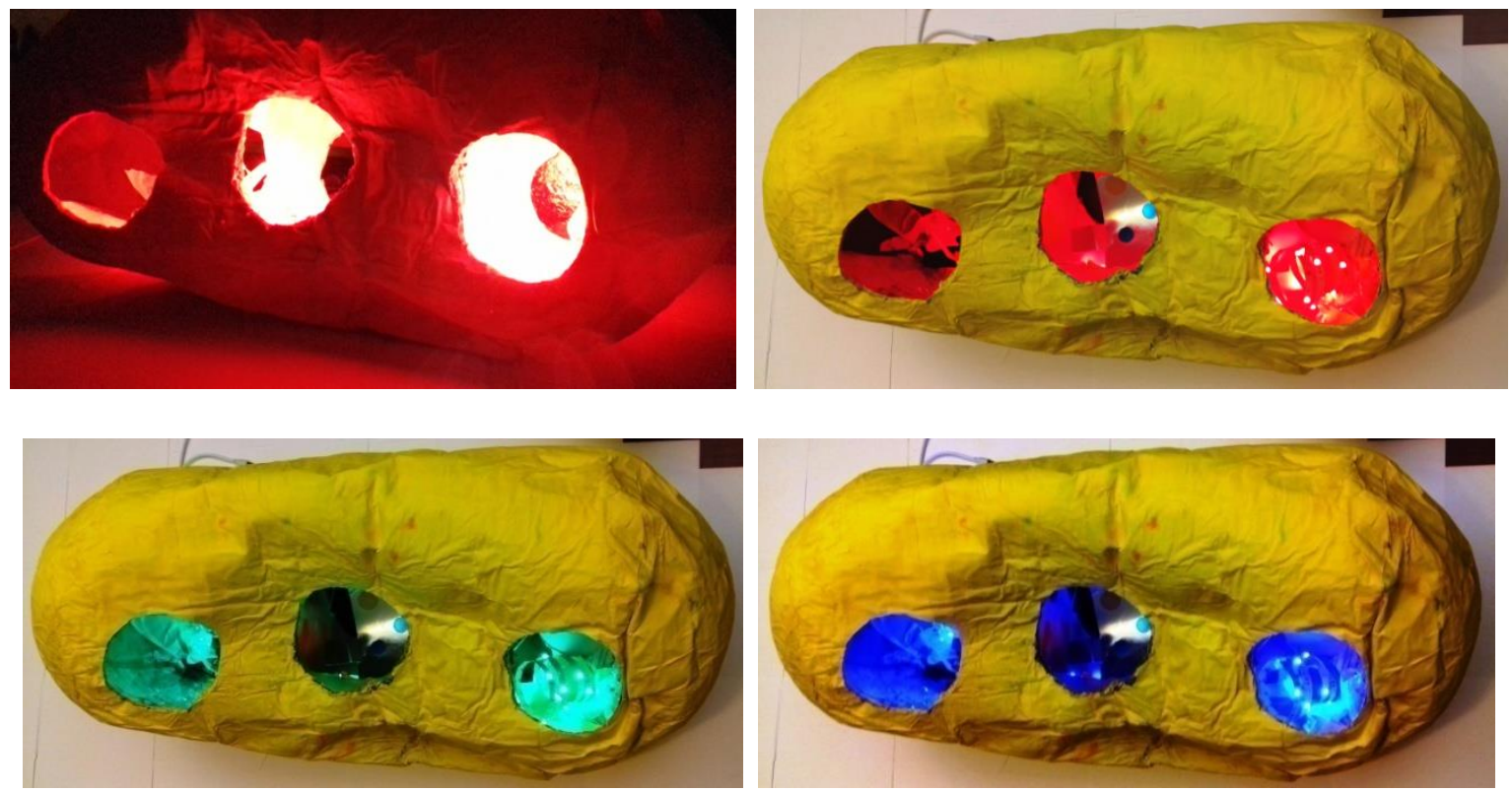

Figure 6: Yellow and Non-Yellow Submarine, handmade paper construction, acrylic colours and LED tape with colorful lights, $80 \mathrm{~cm}$ X $30 \mathrm{~cm}$ X $30 \mathrm{~cm}$.

The last example in Figure 7 involves fruit and vegetable battery puppets with implanted new technologies. The image on the left shows a lemon battery puppet with an implanted motorised fan, while the image on the right shows a potato battery puppet with a LED light nose. This activity is inscribed in STEAM education practices. Discussion was made with regards to chemical and physical phenomena including electric circuits and transformation of chemical energy to electric energy in the case of lemon and potato electrochemical batteries. Lemon and potato become sources of alternative renewable energy and at the same time they can be used in contexts of motivational puppet theatre for children's advice to follow a healthy diet (Synovitz, 1999, Nicklas et al. 2016). Another possibility is to use coding in order to control the time, the duration of lighting, the direction of movement in the case of the fan etc.

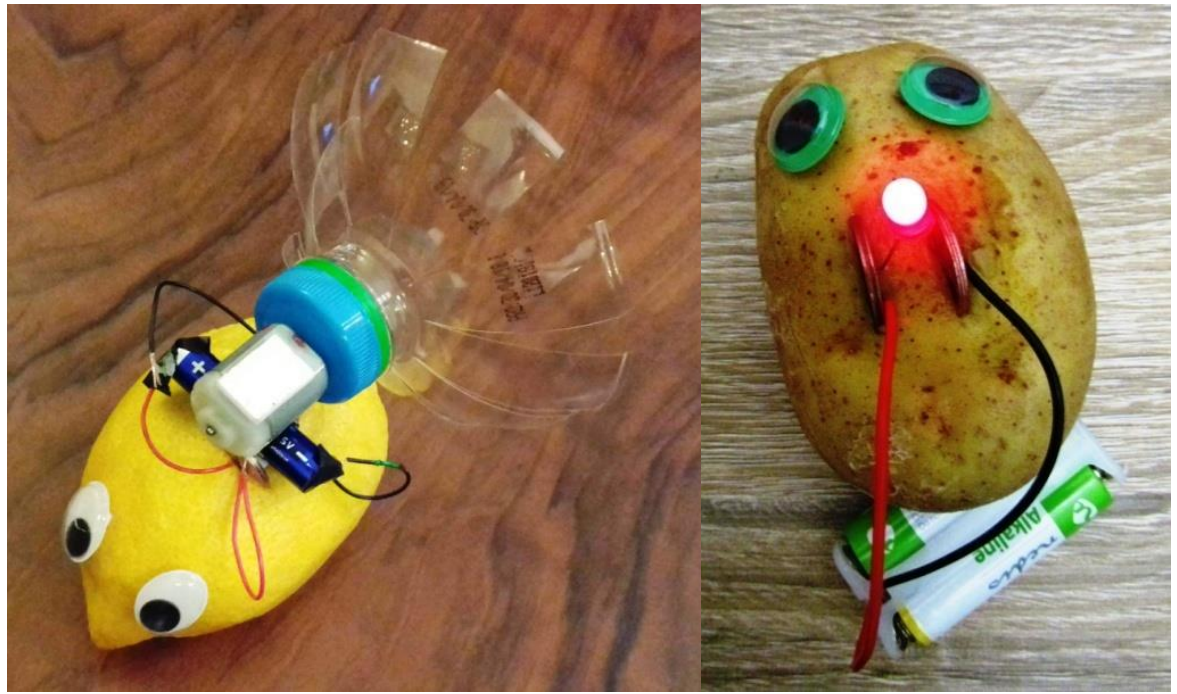

Figure 7: Lemon (left) and potato (right) battery puppets with implanted new technologies: a motorized fan on the lemon and a red LED light nose (right). 


\section{Conclusions}

Although the traditional has largely been opposed to modern and technological (including digital) practices, recent advances in artmaking, art theory, education and everyday life indicate that there is a growing tendency to bring together traditional media and practices with new technologies (Weiming et al. 2010).

Implementation of new technologies in traditional handcrafts leads to redefining the traditional in the contemporary condition and to radically bringing it to the fore, which is the case in the examples of this paper. The force of the examples presented in this paper lies on this very tension between the traditional and the contemporary. The put-togetherness of traditional handcrafts with new technologies bridges the gap between the traditional and the technological, as well as between the traditional and the modern rendering the traditional a cutting-edge contemporary practice far from banalities and out-of-date creation.

"My Fountain was not a negation: I simply tried to create a new idea for an object that everybody thought they knew. Anything can become something else, that is what I wanted to show." Marcel Duchamp, 1961 (Szeeman, 2002)

Furthermore, through the implementation of new technologies in traditional handcrafts, what is being produced is grounded to reality in its using a certain materiality and in its having an inherent reference to certain spatial-temporal and cultural contexts. However, the combination of the traditional with new technologies reveals different aspects and possibilities of the initial object leading to its transformation and providing an extended version of what had been initially perceived as real. This proves that art-and-technology practices which bind the traditional with new technologies put in the spotlight many different aspects of reality by transforming objects, but at the same time by keeping them real.

The need to combine traditional media and techniques with new technologies is inscribed in the contemporary context in which technology has become pre-dominant in our daily lives and, thus, implementation of new technologies in traditional handcrafts ties art-and-technology practices with contemporary society and culture. This need to blur the boundaries between creative practices and society has been of interest for many artists who were preoccupied by the idea of using non-traditional materials (Borchardt-Hume, 2010: 13). However, this paper bridges this gap between the traditional and the technological as well as between the traditional and the contemporary society and culture by incorporating the traditional and by integrating it with new electrical and light technologies.

The implementation of new technologies in traditional handcrafts proves that technology is a unifying platform which fosters artistic, interdisciplinary and (inter)cultural creativity and can be used to promote interdisciplinary and intercultural research, practice and exchange in education.

The tension between the traditional and the contemporary through the coexistence of different media -in the case of the examples from the Higher education presented in this paper- turned out to be a fruitful synergy making the final whole more interesting than the sum of its parts which places the present research in the core of gestalt theory (Koffka, 1935: 649) as well as contemporary system, network and assemblage theories. (Bennett, 2014: 84) 
The implementation of new technologies in traditional handcrafts turned out to be a hands-on art-andtechnology practice with multiple learning outcomes and benefits in education. Students realised that many different aspects of the traditional handcrafts were revealed with the use of new technologies and that their implementation brings traditional handcrafts to (another) life. It also became clear that students' practice through the implementation of new technologies in traditional handcrafts constitutes their own preparation for applying these technologies to children's handmade constructions and, thus, it is possible to enhance and enrich children's creative experience.

The stimulation of many senses thanks to the implementation of new technologies also improves visual and multisensory culture, fosters creativity and contributes to deeper (inter)cultural and technological literacy. Further than merely being passive viewers, consumers and users of contemporary aesthetics and technological culture, participants become creators and active animators of the contemporary aesthetic, artistic and technological multisensory becoming.

This research on implementation of new technologies in traditional handcrafts in the frame of the present article could not be exhaustive, since it provides only seven examples of implementation of electrical and light technologies in traditional handcrafts and this is a considerable limitation. However, the present article sets the basis as a paradigm for further research on similar practices and possible applications in education, special education, (puppet) theatre, stage design, interior and public design as well as everyday life. The practices could be expanded with assemblages made with other kinds of technologies and handcrafts from different traditions and civilizations.

\section{References}

Benjamin, W. (1969) The Work of Art in the Age of Mechanical Reproduction. In: Arendt, H. (Ed.) Illuminations. New York: Schocken Books

Bennett, J. (2014). What is an assemblage? //2010. In: Larsen, L.B. (2014). Networks: Documents of Contemporary Art. (pp. 84-85). London and Cambridge, Massachusetts: Whitechapel Gallery \& The MIT Press

Borchardt-Hume, A. (2010). Keeping it Real: From the ready-made to the everyday. The D. Daskalopoulos Collection. London: Whitechapel Gallery Ventures Limited

Cambridge Dictionary. (2021). Traditional. In Cambridge Dictionary. Retrieved March, 19, 2020, from https://dictionary.cambridge.org/fr/dictionnaire/anglais/traditional

Debord, G. (1958). Rapport sur la construction des situations. Internationale situationniste, Nol

Funk, K. (1999). Technology: Definitions of Technology. Technology and Christian 'values'. Oregon State University - College of Engineering. Retrieved March 19, 2020, from https://web.engr.oregonstate.edu/ funkk/Technology/technology.html

Koffka, K. (1935). Principles of Gestalt Psychology. London: Routledge \& The International Library of Psychology.

Latimer, Q. (2017). [Folio 7] Technology suggests the hand... In: Latimer, Q. \& Szymczyk, A. (Eds.). Documenta 14 Reader. Kassel, Munich, London, New York: documenta und Museum Fridericianum

Merriam-Webster. (n.d.). Traditional. In Merriam-Webster.com dictionary. Retrieved March, 19, 2020, from https://www.merriam-webster.com/dictionary/traditional 
Nicklas, T., Lopez, S., Liu, Y. Reiher, R. (2016). Using Motivational Theatre to Increase Vegetable Consumption by Preschool Children. Journal of the Academy of Nutrition and Dietetics, 116, 9, A35

Stroud, A. \& Baines, L. (2019). Inquiry, Investigative Processes, Art, and Writing in STEAM. In: Khine, M.S. \& Areepattamannil, S. (Eds). STEAM Education: Theory \& Practice. Switzerland: Springer

Synovitz, L.B. (1999), Using Puppetry in a Coordinated School Health Program. Journal of School Health, 69, 145-147. doi:10.1111/j.1746-1561.1999.tb04172.x

Szeeman, H. (2002). Marcel Duchamp. Basel and Ostfildern-Ruit: Museum Jean Tinguely Basel and Hatje Cantz

TATE. (2021). Art term: Appropriation. In TATE online glossary. Retrieved March, 19, 2020, from https://www.tate.org.uk/art/art-terms/a/appropriation

Verwoert, J. (2007). Apropos Appropriation: Why Stealing Images Today Feels Different. Art \& Design Research A Journal of Ideas, Context and Methods, 1 (2), 1, 2/05/2013, Retrieved March 19, 2020, from: http://www.artandresearch.org.uk/v1n2/verwoert.html

Weiming, D., Zhongyang, L., Qian G., (2010). Analysis of the interaction between digital art and traditional art. 2010 International Conference on Networking and Digital Society, Wenzhou, 2010, 441-443.

Wiktionary (2020). Traditional art. In Wiktionary: The free online dictionary. Retrieved March, 19, 2020, from https://en.wiktionary.org/wiki/traditional_art 